\title{
Percutaneous Reconstruction Techniques: Popliteal Artery Approach for Chronic Total Occlusion of Superficial Femoral and Iliac Arteries
}

\section{(1) Emced Khalil}

Ordu University Training and Research Hospital, Clinic of Cardiovascular Surgery, Ordu, Turkey

\begin{abstract}
Objectives: Femoropopliteal artery disease (FPAD) is the most common form of peripheral artery disease. Popliteal artery (PA) puncture enables the use of low-profile sheaths and devices and may be an alternative to the antegrade ipsilateral approach in its treatment. We mainly aimed to discuss the PA retrograde approach with the endovascular treatment (EVT) strategy in FPAD.

Materials and Methods: Twenty patients who underwent EVT with retrograde popliteal approach in superficial femoral artery or PA disease were included in this retrospective study. The decision for retrograde approach was made according to the results of computerized tomography or magnetic resonance angiography. All patients underwent color Doppler ultrasonography at the first and sixth months after intervention. The frequency of procedural complications (hematoma, bleeding, and distal embolism) was recorded.
\end{abstract}

Results: Technical success was achieved in all patients. No transfusions or additional surgical treatments were required in any case. Acute success rate was determined as $100 \%$ blood flow rate assessed by angiography. The patency rates in treated arteries were recorded by ultrasonographic evaluation in the first and sixth months of the post-operative period.

Conclusion: Preoperative evaluation and planning are crucial for the success of interventions in peripheral artery disease. An alternative plan and access site should always be available to ensure success in complex procedures. In light of our findings, retrograde PA puncture can be used safely and effectively in the recanalization of superficial femoral artery and PA stenosis.

Keywords: Superficial femoral artery, endovascular treatment, retrograde

Address for Correspondence: Emced Khalil, Ordu University Training and Research Hospital, Clinic of Cardiovascular Surgery, Ordu, Turkey e-mail: emjedkhalil@gmail.com ORCID: orcid.org/0000-0003-1050-2656

Received: 11.07.2020 Accepted: 12.08.2020

Cite this article as: Khalil E. Percutaneous Reconstruction Techniques: Popliteal Artery Approach for Chronic Total Occlusion of Superficial Femoral and Iliac Arteries. EJCM 2020;8(3):107-112.

DOI: 10.32596/ejcm.galenos.2020.07.035

${ }^{\circ}$ Copyright 2020 by Heart and Health Foundation of Turkey (TÜSAV) / E Journal of Cardiovascular Medicine published by Galenos Publishing House. 


\section{Introduction}

Peripheral artery disease (PAD) is a common cause of cardiovascular morbidity and mortality; in fact, it ranks third after coronary artery disease and stroke $\mathrm{e}^{(1)}$. The elderly population, patients with diabetes mellitus (DM) and smokers have a higher risk of $\mathrm{PAD}^{(2)}$. Most PADs in the lower extremity are caused by atherosclerotic disease, with the superficial femoral artery (SFA) and popliteal artery (PA) being reported as the most common sites $^{(3)}$. Even though up to $50 \%$ of cases are estimated to be asymptomatic, patients with symptoms (especially moderate-severe claudication) often have considerably lower quality of life. It is also established that functional limitations are present in patients without severe symptoms $^{(4)}$. The first-line revascularization strategy recommended in Trans-Atlantic Inter-Society Consensus Document (TASC) II Class D SFA occlusions is identified as femoropopliteal bypass surgery ${ }^{(5)}$.

Extensive lesion size, presence of micro- and macrodissections and vessel diameter adversely affect patency rates after endovascular treatment (EVT). However, in TASC II Class C/D lesions, high success rates are achieved with the advances in EVT methods $(80-90 \%)^{(6)}$. In cases where an antegrade approach is not possible, for instance in cases with SFA proximal or common femoral artery lesions (Figure 1), PA puncture has gained popularity as an alternative, especially with the use of low-profile sheaths and devices ${ }^{(7)}$. Therefore, we aimed to assess the role of retrograde PA approach using the EVT strategy in femoropopliteal artery diseases (FPADs).

\section{Material and Methods}

This retrospective study involved 20 patients who underwent recanalization by retrograde PA approach in EVT due to SFA or PA disease at Ordu University Health Practice and Research Center between 2015 and 2017. The diagnoses of SFA or PA disease were confirmed by magnetic resonance (MR) angiography or computed tomography (CT) angiography in patients with typical symptoms (resting pain, claudication, stenotic peripheral artery disease and cold lower extremities), who also had risk factors for atherosclerotic vascular disease. Patients who had previously undergone SFA or PA interventions, surgery or coronary artery bypass graft surgery were excluded.

The patients were staged according to the Rutherford classification and TASC II classes were also recorded ${ }^{(8,9)}$. The ankle-brachial index (ABI) was calculated before and after surgery and the risk factors were noted ${ }^{(10)}$. All EVT procedures were performed by the same team with the same primary surgeon. The PA puncture site was selected in the same extremity in all patients. Ethic committee approval of the present the study was obtained (decision no: 2020/01 date: 26/02/2020). Written informed consent was obtained from all participants.

Briefly, the patient was given a prone position and, under ultrasonographic guidance, a PA puncture was

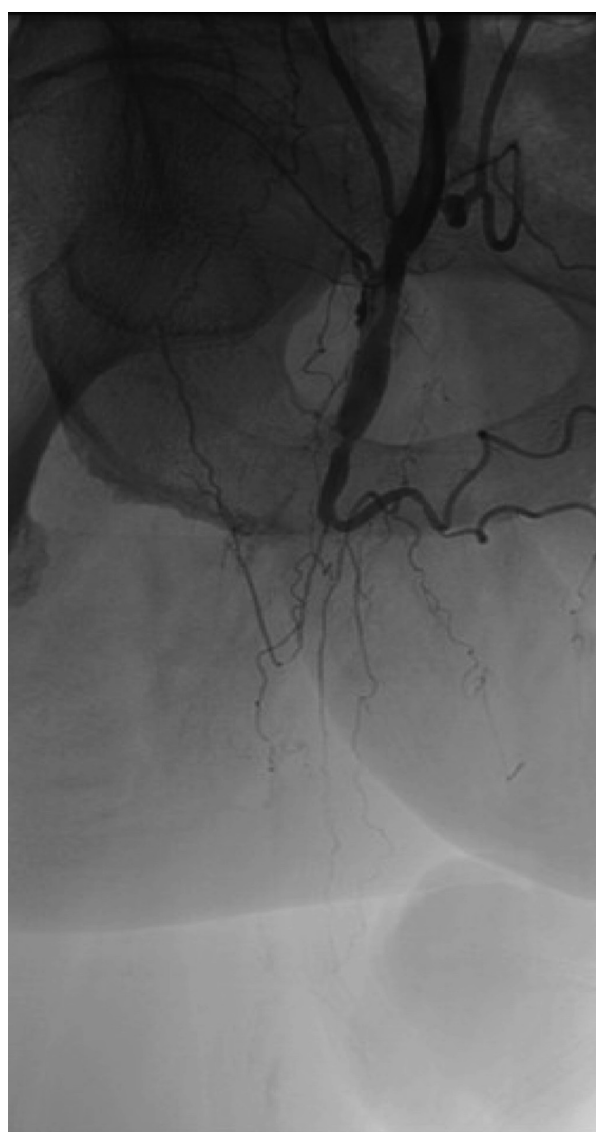

Figure 1. Total occlusion from the common femoral artery 
performed with a 20-22 G needle, and $6 \mathrm{~F}$ sheet was placed (Figure 2A). During the intervention, $100 \mathrm{U} / \mathrm{kg}$ intravenous unfractionated heparin was administered to ensure sufficient anticoagulation. A 0.014 or 0.018 -inch guidewire was advanced into the SFA and PA stenosis region (Figure 2B). Recanalization was performed using Paclitaxel-coated balloons or stents (Figure 2C). After the completion of the procedure, the vascular sheath was removed after being left in the artery for 4 hours after the procedure. During the removal of the sheet, hemostasis was achieved by manual compression. Combined clopidogrel and cilostazol treatment was recommended for 6 months in the post-operative period.

To evaluate the patency of SFA, all patients underwent a physical examination and Doppler ultrasonography (USG) was performed in the first and sixth months of the post-operative period.

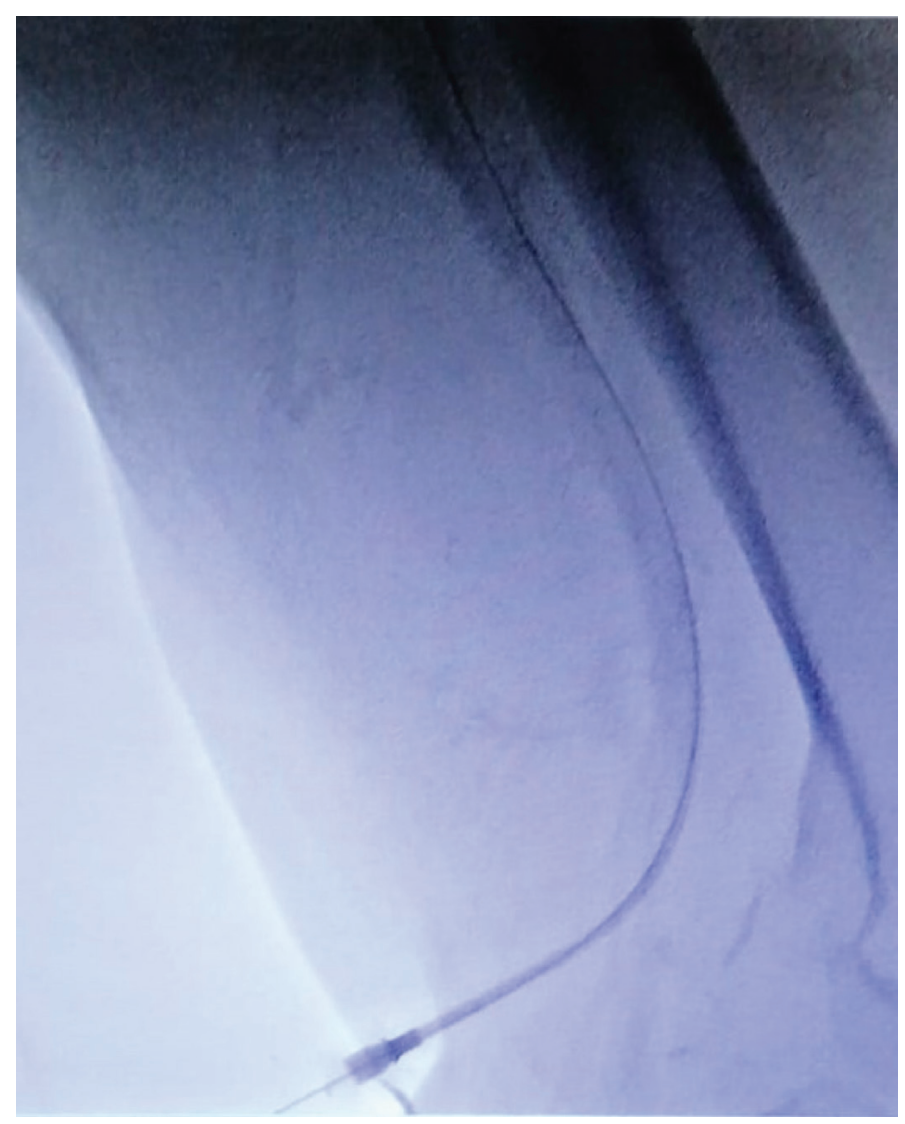

Figure 2A. Sheet placement in the popliteal artery

\section{Statistical Analysis}

All analyses of the study were performed using SPSS (Statistical Package for Social Sciences) for Mac (version 24.0). In the evaluation, number, percentage, mean and standard deviation values were used for descriptive data. The ABI values were given as mean \pm standard deviation values, comparisons were performed with the paired samples t-test. A p value of 0.05 or lower was accepted to be significant.

\section{Results}

The mean age of the 20 patients included in the study was $67.1 \pm 7.1$ years. The demographic and characteristic features of the patients are presented in Table 1. Eightyfive percent of the patients included in the study were male, $60 \%$ had DM, 90\% had hypertension and 35\% were current smokers. Forty percent of the patients were Rutherford category 3, 50\% were category 4 , and $10 \%$

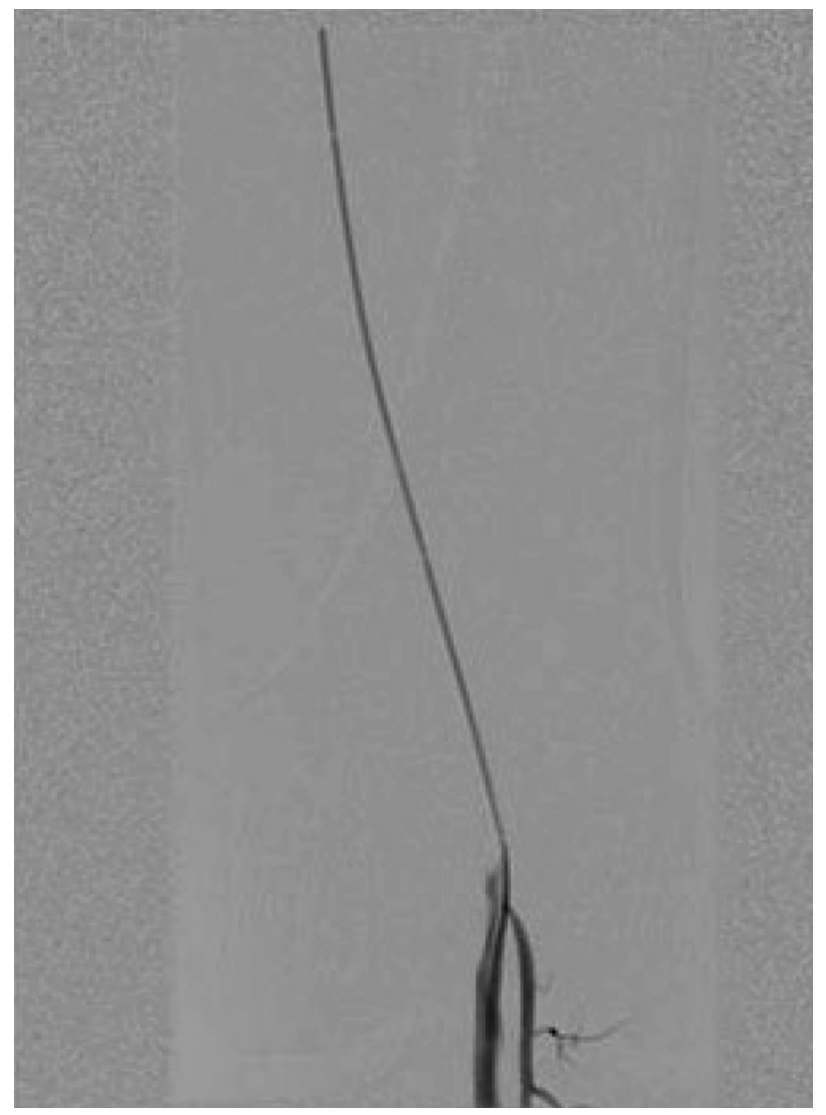

Figure 2B. Advancing the hydrophilic catheter through the lesion 
were category 5. According to the TASC II classification made by evaluating SFA lesions, $50 \%$ of patients were class B, 20\% were class C, and 30\% were class D. The retrograde popliteal approach was successful in all patients. All patients with SFA lesions were symptomatic (claudication and resting pain). Of the interventional complications, bleeding was seen in one patient $(5 \%)$ and hematoma was seen in one patient (5\%). None of the cases that developed intervention complications required blood transfusion or surgical treatment.

After EVT, the success rate of blood flow recovery was determined (by angiography) to be $100 \%$. The ABI

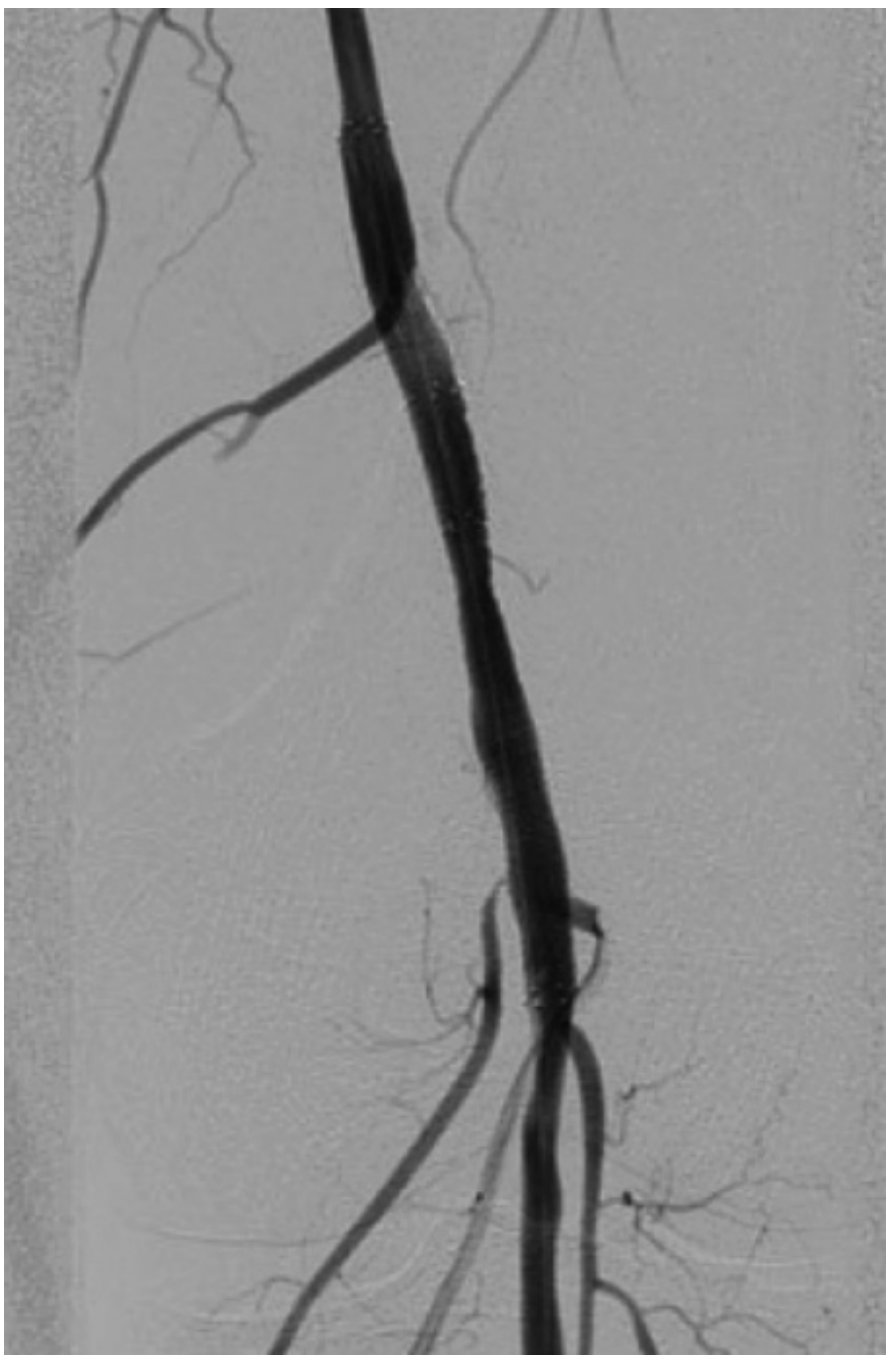

Figure 2C. Image of recanalization flow after drug-coated balloon application values calculated 1 month after the intervention were significantly higher than the ABI values calculated before the intervention (preoperative $=6.72 \pm 0.18$, postoperative $=8.39 \pm 0.09$ ). Patency rates detected via USG on the first and sixth months of the post-operative period were $100 \%$ and $95 \%$, respectively.

\section{Discussion}

According to the results of this single-centered study, EVT with the retrograde popliteal approach is not only

Table 1. Preoperative demographic and clinical features of the cases

\begin{tabular}{|c|c|c|}
\hline & Total (n) & Percentage (\%) \\
\hline Age & 67.10 & \pm SD 7.078 \\
\hline \multicolumn{3}{|l|}{ Gender } \\
\hline Male & 17 & $85 \%$ \\
\hline Female & 3 & $15 \%$ \\
\hline \multicolumn{3}{|l|}{ Risk factors } \\
\hline DM & 12 & $60 \%$ \\
\hline Hypertension & 18 & $90 \%$ \\
\hline Smoking & 7 & $35 \%$ \\
\hline CVD & 6 & $30 \%$ \\
\hline \multicolumn{3}{|l|}{ Complications } \\
\hline None & 18 & $90 \%$ \\
\hline Bleeding & 1 & $5 \%$ \\
\hline Hematoma & 1 & $5 \%$ \\
\hline \multicolumn{3}{|l|}{ Patency } \\
\hline Postop. 1st month & 20 & $100 \%$ \\
\hline Postop. 6th month & 19 & $95 \%$ \\
\hline \multicolumn{3}{|l|}{$\mathrm{ABI}($ mean $\pm \mathrm{SD})$} \\
\hline Preop. & $6.72 \pm 0.18$ & - \\
\hline Postop. & $8.39 \pm 0.09$ & - \\
\hline \multicolumn{3}{|l|}{ Rutherford } \\
\hline 3 & 8 & $40.0 \%$ \\
\hline 4 & 10 & $50 \%$ \\
\hline 5 & 2 & $10 \%$ \\
\hline \multicolumn{3}{|l|}{ TASC II } \\
\hline B & 10 & $50 \%$ \\
\hline C & 4 & $20 \%$ \\
\hline $\mathrm{D}$ & 6 & $30 \%$ \\
\hline \multicolumn{3}{|c|}{$\begin{array}{l}\text { DM: Diabetes mellitus, SD: Standard deviation, CVD: Cardiovascula } \\
\text { disease, Postop: Postoperative, Periop: Perioperative, ABI: The ankle } \\
\text { brachial index, TASC: Trans-Atlantic Inter-Society Consensus Document } \\
\text { n: Number }\end{array}$} \\
\hline
\end{tabular}


successful in distal flow, but also has good patency rates in the short and medium term. This approach had a low complication rate, and those that did develop were minor complications that did not require blood transfusion or surgery. In addition, EVT with the retrograde popliteal approach led to a significant increase in ABI. In the light of these findings, we suggest EVT with the retrograde popliteal approach as an effective and safe approach in complex SFA occlusions.

Although the current study does not report long-term patency findings, it is known that the following factors have major implications for the long-term success of these interventions: patency rate after intervention, the dynamic forces present in these arteries, high calcium levels, and the size of the lesion ${ }^{(11)}$. Surgery is recommended for TASC II $\mathrm{C}$ and $\mathrm{D}$ lesions leading to excessive calcification ${ }^{(12)}$. However, recent evidence also suggests that EVT is applicable in the treatment of $>90 \%$ of femoropopliteal occlusions $^{(13)}$. Most SFA lesions are treated with an antegrade ipsilateral or retrograde contralateral femoral approach. The retrograde popliteal approach was originally considered as a "backup" option but this approach has become the first-choice vascular intervention especially in proximal SFA lesions ${ }^{(9,12,14)}$. The antegrade approach can be difficult to perform in common and proximal femoral artery lesions in the presence of conditions such as narrowing of the aorta or aortic aneurysm ${ }^{(15)}$.

The retrograde popliteal approach was first described in 1988 by Tønnesen et al. ${ }^{(16)}$. Surgeons' increased experience of PA puncture and the use of ultrasonographic and fluoroscopic imaging techniques have increased the reliability and popularity of this approach ${ }^{(17)}$. Data from previous reports suggest that the retrograde popliteal approach may be beneficial in the failure of antegrade recanalization in femoral artery occlusions ${ }^{(18,19)}$. Despite the challenging lesion characteristics, the popliteal approach technique in SFA lesions were reported to have a $100 \%$ success rate in a study that employed 2 years of follow-up ${ }^{(20)}$. In a recent study by Ueshima et al. ${ }^{(7)}$, the success rate of the retrograde popliteal approach in SFA occlusion was $97.2 \%$. A similar success rate was obtained in the treatment of SFA occlusions with the retrograde popliteal approach in the study by Dumantepe ${ }^{(13)}$. In that study, patency rates were $100 \%$ in the first month and $92.8 \%$ in the sixth month of the post-operative period. In a study by Wojtasik-Bakalarz et al. ${ }^{(21)}$, the retrograde popliteal approach was utilized in cases after the failure of anterograde percutaneous recanalization. The patency rate with the retrograde popliteal approach was $88.2 \%$ at the $12^{\text {th }}$ month. In our study, the primary patency rate of patients who underwent a retrograde popliteal approach in SFA lesions was found to be $95 \%$ in the post-operative sixth month.

The popliteal puncture access site appears to be safe in terms of complications. In our study, bleeding occurred in one patient $(2.3 \%)$ and hematoma occurred in one patient $(2.3 \%)$. Both complications were managed with prolonged manual compression. No blood transfusion was required for these patients who had minor complications. Routine USG guidance has been shown in previous studies to reduce complication rate associated with access puncture $^{(22)}$.We believe that $\mathrm{CT}$ or MR angiographic evaluation before the procedures also enables the detailed identification of lesion characteristics and consequently results in less overall exposure to radiation. As such, in the diagnosis of PADs, CT and MR angiography seem to have become a preferred imaging method instead of catheter angiography ${ }^{(23)}$.

\section{Study Limitations}

Even though we report significant success with the retrograde approach as a primary option for the treatment of FPAD, the major disadvantages of our study include its single-centeredness, retrospective nature of data acquisition, and the limited number of patients.

\section{Conclusion}

Preoperative evaluation and planning are critical to achieve success in PAD intervention procedures. An alternative plan and access site should always be available to increase the success of complex procedures. In the 
light of our findings, we believe that the retrograde PA puncture approach can be used safely and effectively in the recanalization of SFA and PA stenosis. This approach should be considered as the primary option, especially in proximal SFA and common femoral artery lesions.

\section{Ethics}

Ethics Committee Approval: Ethic committee approval of the present the study was obtained from Ordu University (decision no: 2020/151 date: 26/02/2020).

Informed Consent: Written informed consent was obtained from all participants.

Peer-review: Internally and externally peer-reviewed.

Financial Disclosure: The authors declared that this study received no financial support.

\section{References}

1. Aronow WS. Peripheral arterial disease of the lower extremities. Arch Med Sci 2012;8:375-88.

2. Kasapis C, Gurm HS. Current approach to the diagnosis and treatment of femoral-popliteal arterial disease. A systematic review. Curr Cardiol Rev 2009;5:296-311.

3. Dhaliwal G, Mukherjee D. Peripheral arterial disease: Epidemiology, natural history, diagnosis and treatment. Int J Angiol 2007;16:36-44.

4. Mcdermott MM, Greenland P, Liu K, et al. Leg symptoms in peripheral arterial disease: associated clinical characteristics and functional impairment. JAMA 2001;286:1599-606.

5. Brountzos EN, Moulakakis KG, Avgerinos ED, et al. Retrograde transpopliteal approach of iliofemoral lesions. Vasc Endovascular Surg 2011;45:646-50.

6. Conrad MF, Cambria RP, Stone DH, et al. Intermediate results of percutaneous endovascular therapy of femoropopliteal occlusive disease: a contemporary series. J Vasc Surg 2006;44:762-9.

7. Ueshima D, Ashikaga T, Shimura T, et al. Popliteal Retrograde Approach is Effective and Safe for Superficial Femoral Artery Chronic Total Occlusion. Ann Vasc Dis 2015;8:220-6.

8. Dormandy JA, Rutherford RB. Management of peripheral arterial disease (PAD). TASC Working Group. TransAtlantic Inter-Society Consensus (TASC). J Vasc Surg 2000;31:1-296.
9. Norgren L, Hiatt WR, Dormandy JA, et al. Inter-society consensus for the management of peripheral arterial disease. Int Angiol 2007;26:81-157.

10. Aboyans V, Criqui MH, Abraham P, et al. Measurement and interpretation of the ankle-brachial index: a scientific statement from the American Heart Association. Circulation 2012;126:2890-909.

11. Stavroulakis K, Argyriou A, Watts M, et al. How to deal with calcium in the superficial femoral artery. J Cardiovasc Surg (Torino) 2019;60:572-81.

12. Goltz JP, Kleemann M. Complex recanalization techniques for complex femoro-popliteal lesions: how to optimize outcomes. J Cardiovasc Surg (Torino) 2015;56:31-41

13. Dumantepe M. Retrograde Popliteal Access to Percutaneous Peripheral Intervention for Chronic Total Occlusion of Superficial Femoral Arteries. Vasc Endovascular Surg 2017;51:240-6.

14. Kawarada O. Commentary. Miniaturized retrograde popliteal approach in a supine patient. J Endovasc Ther 2011;18:510-2.

15. Mahmud E, Cavendish JJ, Salami A. Current Treatment of Peripheral Arterial Disease: Role of Percutaneous Interventional Therapies. J Am Coll Cardiol 2007;50:473-90.

16. Tønnesen KH, Sager P, Karle A, Henriksen L, Jørgensen B. Percutaneous transluminal angioplasty of the superficial femoral artery by retrograde catheterization via the popliteal artery. Cardiovasc Intervent Radiol $1988 ; 11: 127-31$.

17. Schmidt A, Bausback Y, Piorkowski M, et al. Retrograde recanalization technique for use after failed antegrade angioplasty in chronic femoral artery occlusions. J Endovasc Ther 2012;19:23-9.

18. Tan M, Urasawa K, Koshida R, et al. Anterolateral Popliteal Puncture Technique: A Novel Retrograde Approach for Chronic Femoropopliteal Occlusions. J Endovasc Ther 2017;24:525-30.

19. Silvestro M, Palena LM, Manzi M, et al. Anterolateral retrograde access to the distal popliteal artery and to the tibioperoneal trunk for recanalization of femoropopliteal chronic total occlusions. J Vasc Surg 2018;68:1824-32.

20. Khalil E, Çzcan S. Two-Year Follow-Up After Endovascular Therapy of Superficial Femoral Arteries with Retrograde Popliteal Approach: SingleCenter Experience. Heart Surg Forum 2020;23:295-9.

21. Wojtasik-Bakalarz J, Arif S, Chyrchel M, et al. Twelve months follow-up after retrograde recanalization of superficial femoral artery chronic total occlusion. Postepy Kardiol Interwencyjnej 2017;13:47-52.

22. Yilmaz S, Sindel T, Lüleci E. Ultrasound-guided retrograde popliteal artery catheterization: experience in 174 consecutive patients. J Endovasc Ther $2005 ; 12: 714-22$

23. Patel MC, Levin DC, Parker L, Rao VM. Have CT and MR Angiography Replaced Catheter Angiography in Diagnosing Peripheral Arterial Disease? J Am Coll Radiol 2015;12:909-14. 